\title{
Prosthetic arm control using processing device, a comparative approach.
}

\author{
Sudhakar T ${ }^{1}$, Hari Krishnan G $^{2 *}$, Sheeba Santosh ${ }^{3}$, Meenakshi $S^{1}$, Lisha Thomas ${ }^{1}$ \\ ${ }^{1}$ Department of Biomedical Engineering, Sathyabama University, Chennai, Tamil Nadu, India \\ ${ }^{2}$ Department of Electrical and Electronics Engineering, Sree Vidyanikethan Engineering College, Tirupati, Andhra \\ Pradesh, India
}

${ }^{3}$ Department of ECE, Veltech Rangarajan Dr. Sagunthala R\&D Institute of Science \& Technology, India

\begin{abstract}
In this research project, we mainly focus on designing and developing a prosthetic hand using microcontrollers, sensors and feedback for physically challenged. The control and movement of prosthetic hand will be done through the other normally functioning hand. The prosthetic hand will be availed with 2 degrees of freedom, that is, elbow movement, wrist movement and finger movement for pick and place function. The main objective of this project is to provide better working conditions to physically challenged and help them do their day to day activities themselves. Rehabilitation device for arm amputees, that is, an artificial arm which mimics the motion of the normally functioning arm was done with the help of flex sensors and potentiometers.
\end{abstract}

Keywords: Introduction, Rehabilitation, Physically challenged, Amputees, Microcontroller, Sensors, Potentiometers.

Accepted on June 25, 2018

\section{Introduction}

The average prevalence rates in India for males and females are higher in rural areas than in urban areas. But there is a great deal of variation from state to state. In Bihar and Orissa the rates are higher in the urban areas both for males and females. It is not clear why this is so, because higher rates in rural areas would appear to be due to the fact that more persons are involved in manual labor, where the risk of accidents may be higher, and also due to inadequate medical care. Females constitute only 20 percent of the total number of amputees, though they form 48. of the country's population. In the absence of more detailed epidemiological data and information, it is not possible to understand why the ratios are so different for men and women. However, the ratios are not as different in Haryana and rural Rajasthan, where male amputees outnumber women amputees by less than a factor of two. Again, it is not clear why this is so.

The prevalence rates in Punjab and Haryana are the highest: 182 and 244 per 100,000 persons respectively. This may be partly due to amputations caused by threshers and other agricultural machinery introduced in the last fifteen years or so [1]. But this does not seem to be an adequate explanation, as the incidence of paralysis and deformed limbs is also very high in these two states. More epidemiological data are needed to understand why locomotor disabilities should have such a high prevalence rate in Punjab and Haryana. Injuries appear to be one of the major causes of disability accounting for at least 100,000 ( 23 percent) of the amputees. The number is probably larger, as it is possible that many of the amputations due to injury may be hidden under the "other illness" and "other causes" category.

Ron and Rajasekaran developed a robotic arm using raspberry pi. They used internet to control the robotic arm from a remote end. The control signals to robotic arm were provided by the Raspberry Pi and are the cause for arms movements [2]. Shital and Dalvi developed a robotic arm using EMG signals. They used static hand gestures for controlling the robotic arm. Electrodes were used to attain this goal and they took EMG samples from around 10 people to get the rough static gesture values [3]. Aditya and Ridhi reported that a robotic arm can be controlled using EMG signals. They used electrodes to pick up muscle activities in the arm and replicated the motions in the robotic arm. Two electrodes were used and two joint motion was enabled [4]. Shamsheer developed a robotic arm using sensors and microcontrollers. He used ADXL335, a 3-axis accelerometer sensor, L3G4200D, a 3-axis gyroscope sensor, and flex sensor. Since both gyroscope and accelerometer contained high level of noise which makes the values inaccurate, values from both were combined by using Kalman filters [5].

Kevin et al. [6] found a method to control robotic arm virtually. They used BioRadio Electromyography device to capture EMG signals from a human. This signal will undergo modifications such as filtration, amplification and all these are done through National Instruments' LabVIEW graphical programming software. The output is then given to the robotic 
arm. Panagiotis and Kostas developed a robotic arm using low dimensional embeddings. They used EMG signals from muscles of the human upperlimb as the control interface between the user and a robotic arm. A mathematical model was trained to decode upper limb motion from EMG recordings [7]. Minoru et al. developed a robot control system based on electrooculography and electromyogram. They used EOG for moving the robot joint angles and EMG for object grasping. The EOG and EMG discrimination method were used to control the robot [8]. Veena et al. controlled robotic hand with the help of eye movements. Left, right, pick and place movement was enabled. They used five $\mathrm{Ag} / \mathrm{AgCl}$ self-adhesive electrodes for recording the EOG signals [9].

This investigation has a lot of scope for advancement in device that can be modified to assist an amputee with an arm amputed from the shoulders $[9,10]$. The device can be made with wireless connectivity to ease the movement of the artificial arm [11]. Too many wire connections make the movements complex and risky [12]. The sensors can be changed to more precise as well as concise ones, such as MEMS sensors. A number of existing models are there related to robotic arm. Robotic arm that works with remote, EMG signals, EOG signals and many other technologies $[13,14]$.

\section{Materials and Methodologies}

The whole setup is made in a way that, the flex sensors for the wrist and pick and place movement is stuck to a glove and worn on the hand as shown in Figure 1. The potentiometer is placed inside a band with a rod connected to it which enables the potentiometer to rotate.

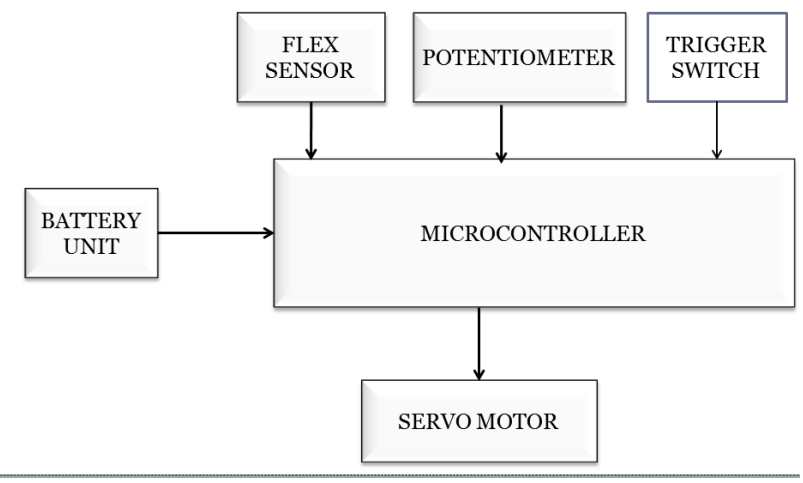

Figure 1. Block diagram of robotic arm.

Several hardware components have been used to fabricate the device and to make it function. The main components are 1. ATMEGA328/P, 2. 7805 IC, 3. 7806 IC 4. Flex sensor and Potentiometer. The Proteus Design Suite is an Electronic Design Automation (EDA) tool including schematic capture, simulation and PCB Layout modules. The Proteus Design Suite is a Windows application for schematic capture, simulation, and PCB layout design. It can be purchased in many configurations, depending on the size of designs being produced and the requirements for microcontroller simulation.

The toggle switch is switched $\mathrm{ON}$ to make the circuit active as shown in Figure 2. The sensors become activated and starts to work. Every motion of the finger is captured by the flex sensor placed on it, which in turn results in the working of the servomotor placed near the gripper. This motion is responsible for the pick and place function. Similarly, the flex sensor placed on the wrist makes the motor responsible for the wrist movement to work. The potentiometer placed on the elbow turns the servomotor placed on the elbow joint of the artificial arm. Flex sensors and the potentiometer are variable resistors. In a flex sensor, the bend of the sensor causes a change in the output resistance which is converted to voltage to make the servomotor work. The same principle is applied in the functioning of the potentiometer.

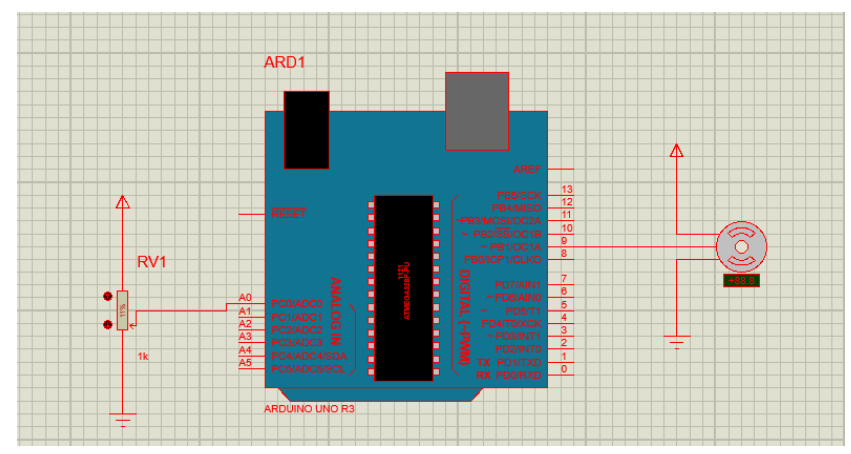

Figure 2. Simulation circuit.

The sensors and the motor are connected to the microcontroller (ATMEGA328). It is a 28 pin IC with 6 analogue input terminals. The sensors are connected to the pins 23, 24 and 25. The servomotors are connected to the pins 15,16 and 17. The outputs from the sensors are mapped and converted into a value between 0 and 180 and given to the servomotor so produce an accurate turn in the motor. The voltage requirement of ATMEGA328 is $5 \mathrm{~V}$. For the servomotors it is $6 \mathrm{~V}$ and for the sensors, $5 \mathrm{~V}$. Due to the requirement of both 5 and $6 \mathrm{~V}$, we use 2 voltage regulators 7805 and 7806 . The output of 7805 is shared by ATMEGA328 and the sensors whereas the output of 7806 is shared by the servomotors.

The device is designed to aid the physically challenged (amputees) by providing them with a robotic arm that can be fitted on the elbow for amputees who have a loss of forearm. The device assists in providing elbow movement, wrist movement as well as pick and place movement, thereby enabling 3 degrees of motion. Figure 3 shows the hardware which comprises of a microcontroller (ATMEGA328), sensors (flex sensors and potentiometer), servomotors, voltage regulators (7805 and 7806) and other components like resistors, crystal, capacitors etc.

The result of the device is tested by running the device. It is found that when the flex sensors move, the pick and place function as well as the wrist movement are properly imitated in the artificial arm. Potentiometer is used for the elbow movement and the motion is accurately mimicked by the artificial arm. Almost every minute movement of the normal arm was successfully mirrored by the robotic arm.

It was also found that the output for the potentiometer is more accurate and stable as compared to that of the flex sensor. The 
discrepancy in the functioning of flex sensor could be due to human error. The functioning of the robotic arm has been found to be $95 \%$ efficient. The $5 \%$ error seen is due to the variations in the output of the flex sensor. Minute errors could also be due to coding discrepancies.

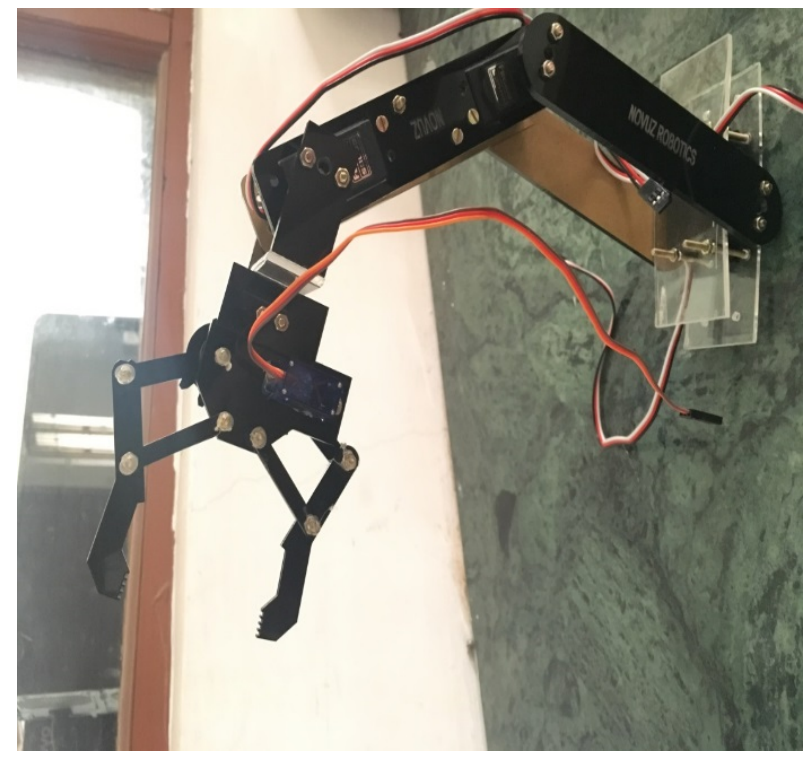

Figure 3. Robotic arm.

Many similar works have been carried out by several researchers in the past [10]. We used ATMEGA328 to interface both sensors and the servo. Abidhusain et al. used an accelerometer together with the flex sensors to check the 3D motion of the arm, in their work [11] observed the flex sensor and accelerometer outputs in graphical form. We have confined to the traditional methods and have used flex sensors and potentiometer alone.

\section{Results and Discussion}

Many previous works include robotic arm that works with EMG, EOG, remote, joystick etc. EMG and EOG signals are found to be highly variable. EMG is obtained from the electrical activity in the muscles and EOG is obtained from the electrical activity in the eyes. Since they are biological signals, their intensity are found to be less and the output requires good amplification. When it comes to remote controlled artificial arm, it's working is highly limited. It just provides a movement function. Vocal controlled robotic arm is also a different field, but the output is limited. It might vary with slight variations in the sound. To obtain a stable output, we chose flex sensor. Flex sensors have a vast number of uses in various fields. The one possible limitation is that, on repeated bending, the stability of the flex sensor output decreases. Flex sensor, in comparison with potentiometer is less stable. Potentiometer output was found to be highly stable with zero noise. But, the limitation faced while working with potentiometer was that, a setup could not be made to fit the potentiometer and to make it rotate according to the hand movement. If this problem can be dealt with, the best desired output can be obtained through a $10 \mathrm{k}$ potentiometer at the least cost.
This research project was mainly aimed at developing a robotic arm for amputees with a loss of arm from the elbow. Two flex sensors and one potentiometer was used to achieve this. The flex sensors were placed on the index finger and the wrist of the normally functioning arm. The potentiometer was placed on the elbow. The movements made in the normally functioning arm were mimicked by robotic arm.

\section{Summary and Conclusion}

The whole work took almost 6 months to complete. Initially, around 10 papers were referred to get ideas about the type of sensors and other components to use. An article on six-servo robotic arm was studied to help in the fabrication of the robotic arm as well as to study the functioning of a $6 \mathrm{~V}$ servomotor. Proteus Design Suite was used to perform simulation to see how the sensors are going to work. After obtaining a positive result from the simulation, trial checks were performed with the sensors to see if the desired outputs are obtained. The hardware was designed and fabricated with 3 servomotors embedded in it to perform the elbow, wrist and pick and place movements.

Voltage regulations were checked multiple times to ensure the safety of the components. The coding was done in Arduino and it was verified multiple times to remove discrepancies. The final interfacing was done to obtain desired result. The flex sensors were stuck on to a glove and worn on one hand. The potentiometer was pasted on a band and worn on the elbow. The whole setup was checked and verified. This robotic hand is very useful for the society as well as industrial application and it works successfully at the time of demonstration. In future it will work on wireless technology with better degrees of freedom.

\section{References}

1. Dinesh M. A report on amputees in India. Orthotics Prosthetics J 1986; 40: 16-32.

2. Ron OT, Rajasekaran K. Remote control of robotic arm using Raspberry Pi. Int J Emerg Technol Comp Sci Electron 2014; 8: 186-188.

3. Shital BS, Dalvi GD. Real time control of robotic arm using Electromyogram (EMG) signals. Int J Sci Res 2014; 5: 797-799.

4. Aditya VSR, Ridhi A. 2-D robotic arm control using EMG signals. Int J Comp Appl 2013; 72: 18-20.

5. Shamsheer V. Hand gestures remote controlled robotic arm. Adv Electron Electr Eng 2013; 3: 602-606.

6. Kevin T, Don G, Jake T. Virtual control of a robotic arm via emg signals processed through labview filter circuit. Sem Schol 2011.

7. Panagiotis KA, Kostas JK. EMG-based control of a robot arm using low-dimensional embeddings. IEEE Trans Robot 2010; 26: 393-398.

8. Minoru S, Muhammad SABS, Kojiro M, Satoshi I, Muhammad IR. Robot control system based on 
electrooculography and electromyogram. J Comp Commun 2015; 3: 114-119.

9. Veena JU, Vaishnodevi S, Mathankumar S. EOG based prosthetic arm-hand control. Int J Innov Res Sci Eng Technol 2015; 5: 3693-3698.

10. Abidhusain S, Zamrrud THA, Thimmannagouday M, Bheemesh G. Flex sensor based robotic arm controller using micro controller. J Software Eng Appl 2012; 5: 12-16.

11. Jayant YH, Niket M, Subodh S, Harshal D. Design for robotic hand using flex-sensor. Int J Adv Res Electron Commun Eng 2015; 4: 130-138.

12. Rutvij B, Mavani Dharmik HR, Helina NS. Design and working of myoelectric prosthetic arm. IJEDR 2014; 2 : 3324-3333.
13. Humaid A, Sayyed J, Maozhen L. Development of Local Prosthetic Limb using Artificial Intelligence. Int J Innov Res Comp Commun Eng 2016; 4: 15708-15716.

14. Richard FW. Design of artificial arms and hands for prosthetic applications. Standard Handbook of Biomedical Engineering and Design 2005.

\section{*Correspondence to}

Hari Krishnan G

Department of Electrical and Electronics Engineering Sree Vidyanikethan Engineering College

India 